\title{
Orbital Metastasis An Analysis of Nine Cases And Review of Literature
}

\author{
Kamlesh Singh Bhaisora ${ }^{1}$,Rabi Narayan Sahu ${ }^{1}$,Amit Kumar Singh ${ }^{1}$, \\ Ruchi Tomer ${ }^{2}$,Pradeep Sharma ${ }^{1}$,Jayesh Shardhara ${ }^{1}$ Vikas Kanaujia $^{2}$, \\ 1.Kuntal Kanti Das ${ }^{1}$,Anant Mehrotra ${ }^{1}$,Arun Kumar Srivastava ${ }^{1}$, \\ Awadhesh Kumar Jaiswal ${ }^{1}$, Sanjay Behari ${ }^{1}$ \\ ${ }^{I}$ (Department of Neurosurgery, Sanjay Gandhi Postgraduate Institute of Medical Sciences, lucknow, India) \\ ${ }^{2}$ (Department of Opthalmology, Sanjay Gandhi Postgraduate Institute of Medical Sciences, lucknow, India)
}

\begin{abstract}
:
Aim: To analyze nine cases of orbital metastasis with review the literature for incidence, presentation, evaluation and discuss their multidisciplinary management.

Materials and Methods: Retrospective study of nine patients between Jan 2012-March 2015 Results: Out of nine consecutive patients with orbital metastasis treated in three year at the department of neurosurgery and neuro-ophthalmology, six patients (75\%) were 60 years or older and two were less than 60 years at diagnosis. M: $F$ ratio was $2: 1 \quad(M=6, F=3)$. These patients presented with proptosis in eight (88.8\%), pain in four (44.4\%), restriction of eye movement in six (66.6\%) patients and vision loss in two (22.2\%). All these patients presented primarily with orbital symptoms without known primary tumor. Metastatic adeno carcinoma $(n=4)$ was most common histology followed by adenoid cystic carcinoma of salivary gland $(n=2)$, malignant lymphoma, papillary carcinoma of thyroid and malignant osteosarcoma was present in 1 patient each.Over a mean follow-up of eighteen months, seven (77.7\%) patients had orbital wall metastasis related systemic involvement and one case (11.1\%) remained free of systemic involvement after chemo. for lymphoma. Three (33.3\%) patients had recurrence following surgery within six months.

Conclusions: Orbital metastasis is rare and in 1/3 of patients presents as initial presentation with unknown primary. Gastro-intestinal tract, lungs, breast and kidney are most common cancers frequently metastases to orbit. Management of orbital metastasis depends on the outcome of primary disease and overall clinical condition of the patient. The prognosis of these tumors is variable depending on the tissue diagnosis.

Keywords: orbit, metastasis, secondary, proptosis
\end{abstract}

\section{Introduction}

Orbital metastasis is an uncommon cause of proptosis; various literatures has documented 3-6\% incidence. [1] These metastatic deposits may precede symptoms of primary in many cases like in lung, gastrointestinal, thyroid and renal cancers.[2,3]Some cases of carcinoma breast present with orbital metastasis after completing treatment for the primary.[2,3] Now a day evaluation for unknown primary in case of metastases is relatively easy with advancement in technology. Nevertheless, in some cases the primary remains unknown until the tumor present as metastasis elsewhere. In literature incidence of unknown primary has been reported upto $2 \%$ of all oncology reference.[4] Fizazi et al have reported incidence of metastasis with unknown primary between $3-5 \%$.[5] In cases which are suspicious for metastasis on clinical and radiological evaluation; fine needle biopsy can be done to confirm in preop period. In most cases identification of primary do not alter overall outcome but some survival advantage can be seen in lymphoma, breast carcinoma and uterine malignancies. [3, 6] In this study we present our experiences of orbital metastasis in nine patients with review of literature regarding their presentation and prognosis. All these patients presented to our department primarily with orbital symptoms with unknown primary.

\section{Materials and Methods}

This is a retrospective study of nine cases of orbital metastasis, treated at our center in three years period between Jan 2012 to March 2015. All clinical, radiological and histopathology records of these patients were reviewed. All these patients underwent surgical excision with post-op adjuvant therapy, complete evaluation for identification of primary site done in all patients

III. Results 
Out of nine consecutive patients with orbital metastasis treated in three year at the department of neurosurgery and neuro-ophthalmology, six patients $(66.6 \%)$ were 60 years or older and three $(33.3 \%)$ were less than 60 years at diagnosis. There were six $(66.6 \%)$ male and three $(33.3 \%)$ female patients in our group. The common clinical features at presentation was swelling with proptosis in eight (88.8\%), and pain in four (44.4\%) and restriction of eye movement in six $(66.6 \%)$ patients and vision loss in two $(22.2 \%)$ cases. Mean duration of symptoms was 5.2 months before referral. The histology was metastatic adeno-carcinoma in four patients (44.4\%), adenoid cystic carcinoma of salivary gland in two patients (22.2\%), malignant lymphoma, osteosarcoma and papillary carcinoma in one patient each $(11.1 \%)$.

The diagnosis was made with clinical findings, imaging studies and histopathology after surgical excision in all patients. Main tumor location was in the superior orbito-frontal wall in two patients $(22.2 \%)$, one in the medial wall of the orbit $(11.1 \%)$, and in the lateral wall of the bony orbit in six cases $(66.6 \%)$ (Figure 1a,1b). Mean size of the tumor was $5.3 \mathrm{~cm}$, with the most of larger tumors found in the lateral wall side. Small sized lateral wall tumor were approach by lateral orbitotomy, lateral wall tumors which were larger in size with sub frontal extension were excised with pterional extradural approach (Figure 2a,2b,2c). Pterional extradural approach is ideal for decompression of optic nerve in optic canal and for tumor near superior and inferior orbital fissure and cavernous sinus. Sub-frontal approach was taken for tumor involving superior orbito-frontal wall. Endoscopic endonasal approach was used for removing tumor from the medial orbital wall in one patient. Drill was used in cases with hard or firm tumors involving bone; excision of dura with lax duraplasty done with fascia lata in cases where dura found to be involved. Tumor was solid to firm in six cases (66.6\%), soft to cystic in three cases $(33.3 \%)$. Over a mean follow-up of eighteen months, seven $(77.7 \%)$ patients had orbital wall metastasis related systemic involvement and one case $(11.1 \%)$ remained free of systemic involvement after chemotherapy for lymphoma. One patient with histology of papillary carcinoma thyroid; undergone evaluation for thyroid malignancy and planned for surgery for primary disease. Three (33.3\%) patients had recurrence following surgery within six months.

Figures 1

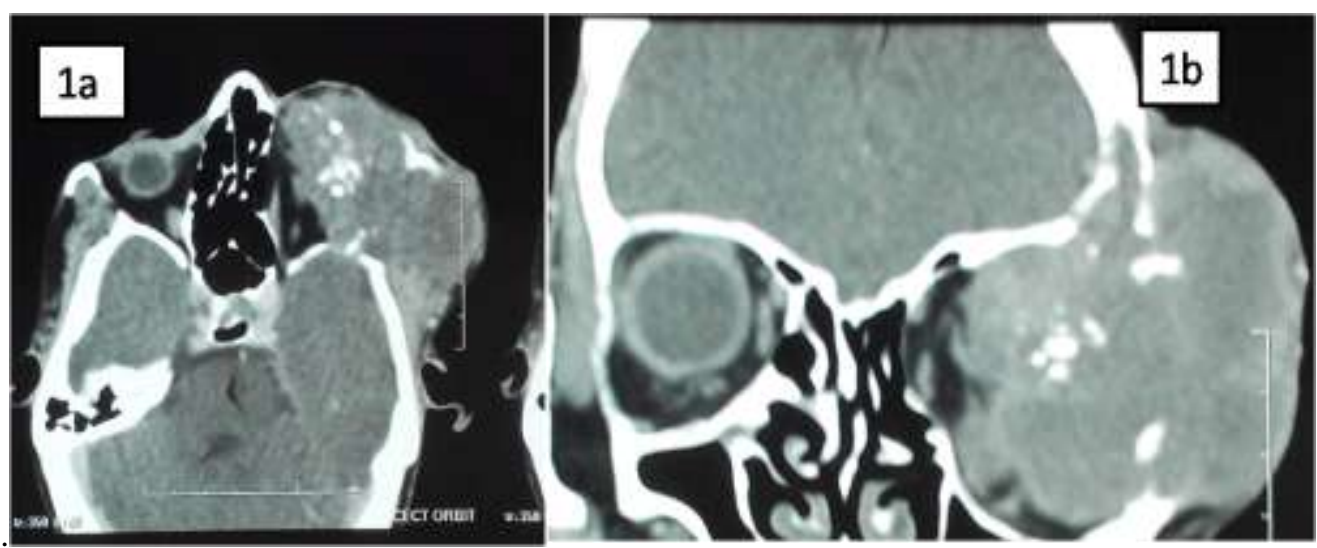

Axial (1a) and coronal (1b) of cuts contrast enhanced CT head and orbit showing heterogeneous minimal contrast enhancing mass involving left lateral orbital wall displacing eye ball anteriorly with lump on temporal side of head.

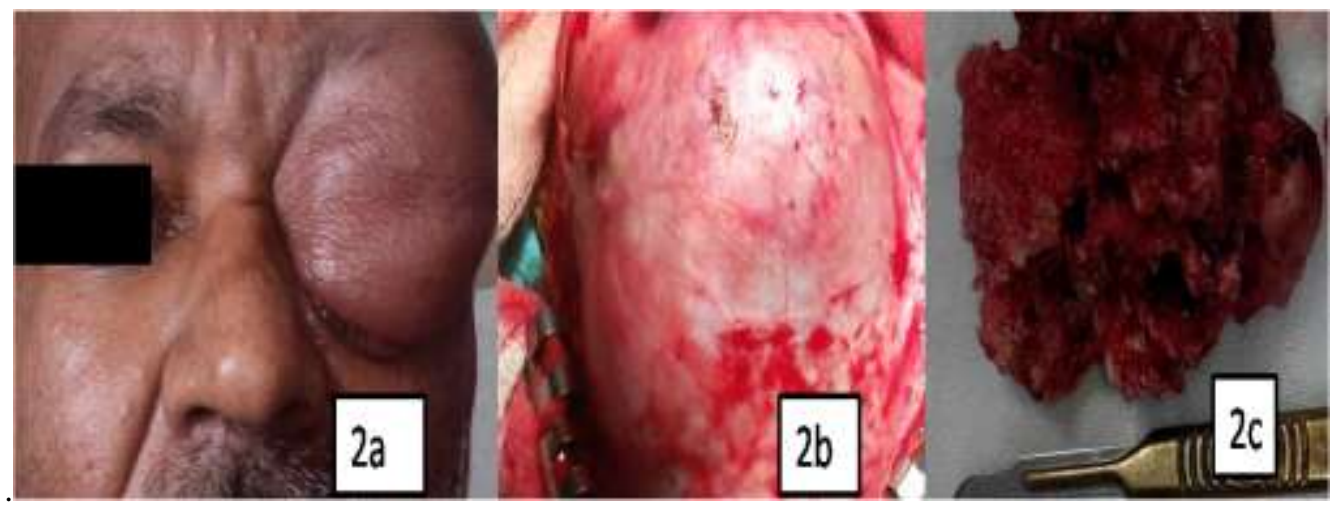

Presurgery photograph of patient having left eye proptosis due to orbital tumor(2a), intraoperative photograph during tumor exposure by pterional incision (2b), excised tumor (2c) 


\section{Discussion}

Orbital metastasis is an uncommon condition in literature, it has been reported 2-7\% of all orbital tumors.[1] Due to advancement in imaging more cases of orbital metastasis being reported, this may be due to increased survival of these patients due to newer targeted therapies and better imaging tools. Most common mode of spread of this metastasis is hematogenous route. Ocular metastasis is more common than orbital metastasis, with 1: 8 ratios in histopathological series.[1,7] Most common site for orbital metastasis is extraconal $(50 \%)$ but as tumor grows in size they usually involve both intraconal and extraconal compartment.[8] Bilateral involvement is more common with intraocular metastasis; various studies have reported $25 \%$ cases had bilateral tumors. Bilaterality is not common in orbital metastasis. In our study there was no patient with bilateral involvement. Tijl et al in their study reported 4 of 34 patients had bilateral involvement and all of these patients had breast carcinoma.[9] One third of these patients present without having history of primary tumor.[1] Front and Ferry, in a series reported from national pathology registry, noted that 17 of 28 patients $(61 \%)$ presented with orbital metastasis with unknown primary.[1,2]

Most common causes of metastasis to orbit are breast, prostate, lung and kidney; they make 60-70\% of all cases metastasizing to orbit; other causes of orbital metastasis have been reported in various tumor located at different sites such as skin, stomach, bone, pleura, gall bladder and testis.[1,3,6] In our study four patients (44.4\%) had adenocarcinoma, two patients $(22.2 \%)$ had adenoid cystic carcinoma of salivary glands and one patient $(11.1 \%)$ each had malignant lymphoma, osteosarcoma and papillary carcinoma thyroid in histopathology.

Most common site in orbit is lateral (39\%) and superior wall (32\%) than medial (20\%) and inferior wall $(12 \%)$ has been reported in literature. [3,7] Metastasis from different tumors involves different tissue preferentially, to start with like prostatic metastasis involves bone initially, Breast metastasis involve fatty tissue and muscle; melanoma involves preferentially muscle. Overall distribution of metastasis in orbit seems to be in; 2:2:1 bone- fat- muscle ratio.[3,7] In our series lateral wall involvement was seen in six patients $(66.6 \%)$, superior orbito-frontal wall in 2 patients $(22 ; 2 \%)$ and medial wall involvement was seen in one patient $(11.1 \%)$. In pediatric patients sarcomas are more common than carcinoma with neuroblastoma and Ewing's sarcomas most commonly metastasizing tumors.[7]

Patients with orbital metastasis primarily consult ophthalmologist with complains of diplopia, pain, diminution of vision, proptosis or ophthalamoplegia. Most common presentation is restriction of orbital motility and proptosis. Tumor which involved muscle first will cause restriction of eye movement to start with and other extraconal mass will present as proptosis. Diminution of vision one of the most common cause for surgical intervention with occur due to compression of optic nerve. Involvement of fifth nerve branches may cause sensory hypoasthesia over face. Bony metastasis which causes erosion of orbital roof may present with pulsatile proptosis due to direct transmission of intracranial pressure to orbit. Thyroid and renal tumors may cause pulsatile proptosis due their hypervascularity. Scirrhous carcinoma of breast may cause enophthalmos than proptosis due tendency to cause fibrosis. Goldberg et al. suggested categorization into five generalized syndromes of presentation, frequencies of which were as follows: infiltrative (53\%); mass (37\%); inflammatory (5\%); functional (3\%); and silent.[3,6]

CT orbit and MR of orbit commonly used for evaluation of these patients. CT orbit is best for evaluation of orbital pathology but intraorbital soft tissue component may be evaluated better with MRI. Tumors which are extensive; MRI brain should be included in study to look for intracranial extension. Rarely these lesions may simulate nonmalignant lesion like hemangioma, myositis, thyroid ophthalamopathy or other inflammatory condition. Fine needle biopsy may beneficial in identifying pathology of tumors with unknown primary; according to which primary can be investigated. [1, 10]

Treatment of orbital metastasis depends upon correct diagnosis, systemic status of patient and optic nerve compression.Surgery advocated in cases who presents with symptoms of optic nerve compression in good systemic status, with feature of intracranial extension with mass effect and tumors causing painful disfiguring proptosis. Surgical excision apart from giving a local disease control also gives a tissue diagnosis for searching the primary site. Patients with diffuse solitary orbital tumor with poor systemic status can be managed with direct radiotherapy, $80 \%$ of patients will have symptomatic relief.[1] Hormonal therapy plays an important role in the treatment of hormone sensitive tumors like breast cancer, some meningiomas and prostate cancers.[1,11] Systemic chemotherapy found to be helpful in metastasis from tumors like small cell lung cancer and neuroblastoma.[1] Ratanatharathorn et al in their study found that in 32 patients with ocular and orbital breast metastasis 10 patients had secondary in brain. So these patients should be evaluated for synchronous metastasis so that if continuous metastatic deposits present; they can be included in same treatment plan during radiotherapy.[12] 
Prognosis of these patients depends upon type of the primary tumor, treatment-response of primary, systemic involvement by tumor and clinical condition of patients. Char et al have shown 1.3 yrs of overall survival in their series with $27 \%$ cases survived for 2 years.[1] Another series have shown overall survival of 9.3 months for their patients. In our study after mean follow up of 16.33 months (Range 3- 18 months) 8 patients had no recurrence at orbit but had feature of systemic involvement, 1 patient of lymphoma was free from systemic disease after chemotherapy and three patients had recurrence of orbital metastasis.

\section{Conclusion}

Orbital wall metastasis is rare and in one third of patients, orbital symptoms are initial presentation. These patients usually presents with pain, diplopia, diminution of vision, restriction of eye movements and proptosis; rarely may present with mass effect when intracranial extension is present. CT scan of orbit and contrast MRI imaging is required for evaluation in pre-op period. Patients with orbital metastasis with compressive features like optic nerve compression, oculo-paresis and painful disfiguring proptosis are treated with wide surgical excision followed by radiotherapy, chemotherapy and/or hormonal therapy. Surgery for orbital metastasis; apart from giving a local cyto-reduction control also provides a tissue diagnosis for searching the primary tumor. The prognosis of the orbital metastases is variable depending on the histological diagnosis and treatment-response of the primary pathology. Some cases with hematological malignancies like lymphoma / plasmacytoma have better prognosis than with cases of adeno-carcinoma.

\section{Acknowledgements: Nil}

\section{References}

[1]. Char DH, Miller T, Kroll S. Orbital metastasis: diagnosis and course. Br J Ophthalmol 1997;81:386-390.

[2]. Font RL, Ferry AP. Carcinoma metastatic to the eye and orbit . A clinicopathologic study of 28 cases metastatic to the orbit. Cancer 1976;38:1326 35 .

[3]. Goldberg RA, Rootman J, Cline R. Tumors metastatic to the orbit: a changing picture. Surv Ophthal 1990;35:1-24.

[4]. Abbruzzese JL, Abbruzzese MC, Lenzi R, Hess KR, Raber MN. Analysis of a diagnostic strategy for patients with suspected tumors of unknown origin. J Clin Oncol 1995;13:2094-103.

[5]. Fizazi K,. Greco FA, Pavlidis N, Pentheroudakis G. Cancers of unknown primary site: ESMO Clinical Practice Guidelines for diagnosis, treatment and follow-up. Annals of Oncology 2011; 22:64-68.

[6]. Goldberg RA, Rootman J. Clinical characteristics of metastatic orbital tumors. Ophthalmology 1990; 97:620-624.

[7]. Ahmad SM, Esmaeli B. Metastatic tumors of the orbit and ocular adnexa. Curr Opin Ophthalmol. 2007;18:405-13

[8]. Gonzalez F, Lopez-Couto C. Orbital metastasis: A report of four cases and a review of the literature. Arch Soc Esp Oftalmol. 2006;81:451-62.

[9]. Tijl J, Koornneef L, Eijpe A, Thomas L, Gonzales DG, Beenhof C. Metastatic tumors to the orbit-management and prognosis. Graefes Arch Clin Exp Ophthalmol 1992;230:527-30.

[10]. Gupta S, Sood B, Gulati M, Takhtani D, Bapuraj R, Khandelwal N, et al. Orbital mass lesions: US-guided fineneedle aspiration biopsy - experience in 37 patients. Radiology 1999; 213: 568-572.

[11]. Eckardt A M, Rana M, Essig H, Gellrich N. Orbital metastases as first sign of metastatic spread in breast cancer: Case report and review of the literature. Head \& Neck Oncology 2011; 3:37.

[12]. Ratanatharathorn V, Powers WE, Grimm J, Steverson N,Han I, Ahmad K, et al. Eye metastasis from carcinoma of the breast: diagnosis, radiation treatment and results. Cancer Treat Rev 1991;18:261-76. 